\title{
Resolving complex chromosome structures during meiosis: versatile deployment of Smc5/6
}

\author{
Dideke E. Verver $^{1}$ • Grace H. Hwang ${ }^{2}$ Philip W. Jordan ${ }^{2}$ • Geert Hamer ${ }^{1}$
}

Received: 11 February 2015 /Revised: 2 April 2015 / Accepted: 20 April 2015 / Published online: 7 May 2015

(C) The Author(s) 2015. This article is published with open access at Springerlink.com

\begin{abstract}
The Smc5/6 complex, along with cohesin and condensin, is a member of the structural maintenance of chromosome (SMC) family, large ring-like protein complexes that are essential for chromatin structure and function. Thanks to numerous studies of the mitotic cell cycle, Smc5/6 has been implicated to have roles in homologous recombination, restart of stalled replication forks, maintenance of ribosomal DNA (rDNA) and heterochromatin, telomerase-independent telomere elongation, and regulation of chromosome topology. The nature of these functions implies that the Smc $5 / 6$ complex also contributes to the profound chromatin changes, including meiotic recombination, that characterize meiosis. Only recently, studies in diverse model organisms have focused on the potential meiotic roles of the Smc5/6 complex. Indeed, Smc5/ 6 appears to be essential for meiotic recombination. However, due to both the complexity of the process of meiosis and the versatility of the Smc5/6 complex, many additional meiotic functions have been described. In this review, we provide a clear overview of the multiple functions found so far for the Smc5/6 complex in meiosis. Additionally, we compare these meiotic functions with the known mitotic functions in an
\end{abstract}

Dideke E. Verver and Grace H. Hwang contributted equally to this work.

Philip W. Jordan

pjordan8@jhu.edu

Geert Hamer

g.hamer@amc.uva.nl

1 Center for Reproductive Medicine, Women's and Children's Hospital, Academic Medical Center, University of Amsterdam, Amsterdam, The Netherlands

2 Department of Biochemistry and Molecular Biology, Johns Hopkins University Bloomberg School of Public Health, Baltimore, MD, USA attempt to find a common denominator and thereby create clarity in the field of Smc5/6 research.

Keywords Structural maintenance of chromosomes . Smc5/6 $\cdot$ Meiosis $\cdot$ Meiotic recombination $\cdot$ DNA repair

\section{Smc5/6 complex structure}

The Smc5/6 complex is a member of the structural maintenance of chromosome (SMC) family, along with cohesin and condensin. The Smc5/6 complex is proposed to have the characteristic ring-like structure of the SMC family in which each SMC complex is comprised of two SMC proteins forming a heterodimer and multiple non-SMC elements (reviewed in (Jeppsson et al. 2014b)). The Smc5/6 complex is comprised of Smc5, Smc6, and several non-SMC elements of which Nse1-4 are conserved from yeast (Duan et al. 2009; Hazbun et al. 2003; Pebernard et al. 2006; Zhao and Blobel 2005) (Fig. 1a, b) to mammals (De Piccoli et al. 2009; Taylor et al. 2008) (Fig. 1c). When referring to the Smc $5 / 6$ complex genes or proteins in general, we will use yeast nomenclature. When referring to a specific organism, or data obtained using a specific organism, we will use the specific nomenclature of that organism, e.g., NSMCE1 for the mammalian ortholog of Nse1. The SMC proteins have an extensive coiled-coil domain interrupted by a hinge domain that folds each SMC back on itself. The two globular $\mathrm{C}$ and $\mathrm{N}$ terminal ends are juxtaposed to form an ATP-binding and ATP-hydrolysis site (Fig. 1d). To form a closed-ring structure, the ATPase domains are bridged together by non-SMC elements, while the SMC proteins associate tightly through their hinge regions (reviewed in (Jeppsson et al. 2014b)).

In vitro assays using purified fission yeast proteins have shown that Nse1 binds to Nse3, and both Nse1 and Nse3 bind 
Fig. 1 Structure and composition of Smc5/6 complex. Conserved from yeast to humans, Smc5 and Smc6 fold and interact at their central hinge domains. Through the coiled-coil stretch, the $\mathrm{N}$ - and C-termini are brought in close proximity creating an ATPase domain. The ring-like structure is closed by several non-SMC elements (Nse1, Nse3, and Nse4). In addition, the SUMO ligase Nse2 is bound to the coiled-coil region of Smc5. Nse5 and Nse6 are located at the hinge domain in budding yeast (a), at the ATPase domain in fission yeast (b), but homologs have not been identified in mammals (c). d Smc5 and Smc6 each contain an extensive coiled-coil domain that folds back on itself at a central hinge domain, juxtaposing the terminal head domains to form an ATP-binding and ATP-hydrolysis site
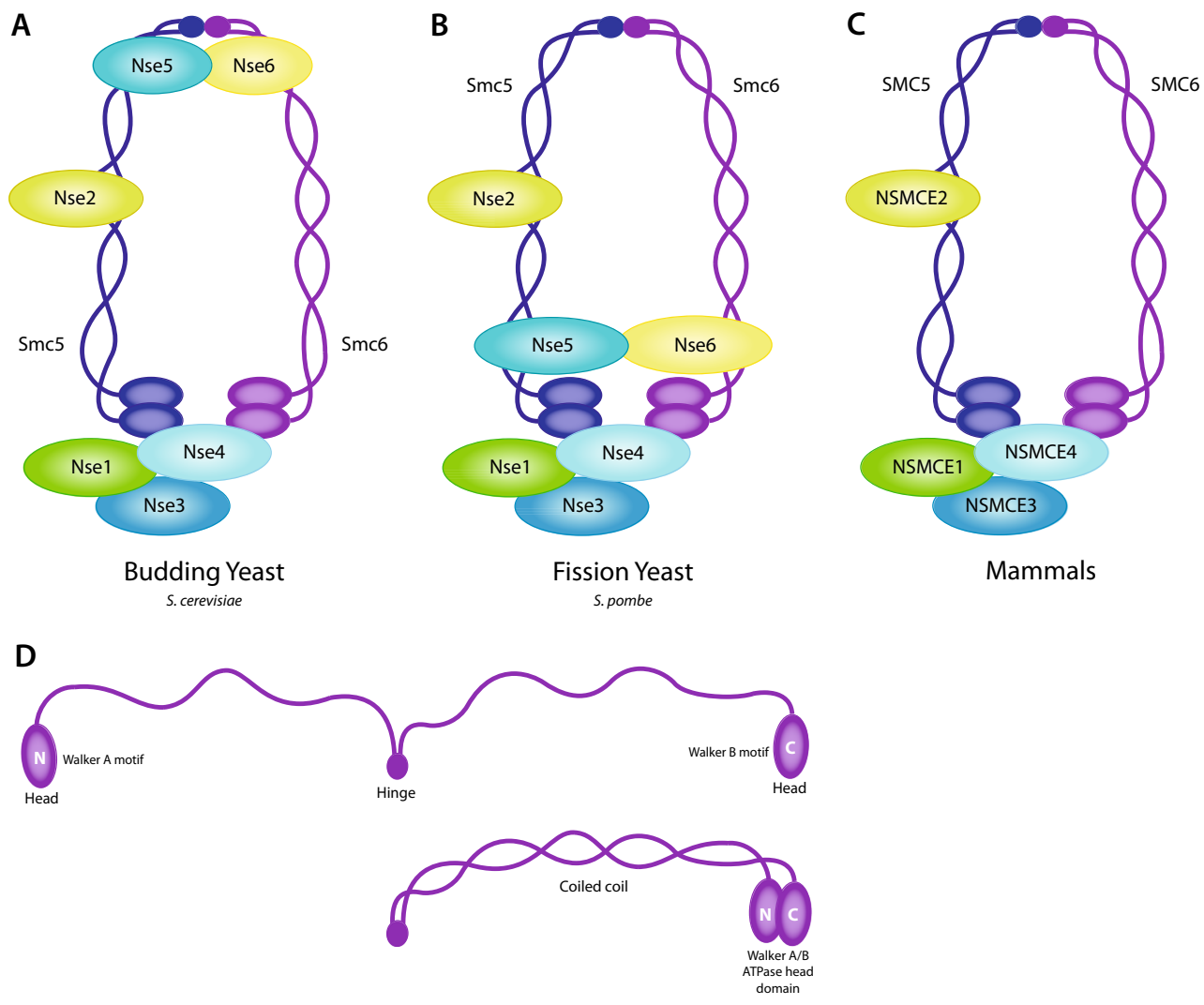

to Nse4 (Palecek et al. 2006; Pebernard et al. 2008). Nse1 contains a RING-finger domain, common to ubiquitin E3 ligases (Fujioka et al. 2002; McDonald et al. 2003; Potts 2009), and Nse3 contains a MAGE (melanoma-associated antigen gene) domain (Pebernard et al. 2004). It has been shown that human NSMCE3 enhances the E3 ubiquitin ligase of NSMC E1 in vitro (Doyle et al. 2010). Nse2 (also referred to as Mms21) is bound to Smc5, contains a SP-RING domain (McDonald et al. 2003; Pebernard et al. 2004), and functions as an E3 small ubiquitin-related modifier (SUMO) ligase (Andrews et al. 2005; Potts and Yu 2007; Zhao and Blobel 2005). Nse4 is a $\alpha$-kleisin subunit which bridges the ATPase head domains of Smc5 and Smc6 (Palecek et al. 2006). Nse5 and Nse6 are also Smc5/6 components in budding and fission yeast, although homologs in other organisms have not been elucidated. In budding yeast, Nse5 and Nse6 associate with the hinge region (Fig. 1a) (Duan et al. 2009). In fission yeast, Nse5 and Nse6 associate with the head domains (Fig. 1b), which may enhance the stability of the complex (Pebernard et al. 2006).

\section{Smc5/6 in mitotic cells}

In somatic cells, the Smc5/6 complex is involved in several processes required to maintain genomic stability. Mechanistically, these processes involve regulation of specific factors required for homologous recombination (HR) pathways. All these processes, including DNA replication, HRmediated DNA double strand break (DSB) repair, correct chromosome topology and, eventually, proper metaphase conformation, are also essential for successful meiosis.

\section{Smc5/6 and stalled replication forks}

Smc5/6 is required for maintaining replication fork stability and the restart of stalled replication. In budding yeast, the absence of Nse2 SUMO ligase activity results in Rad51dependent X-shaped HR intermediates or aberrant joint molecules (JMs) accumulating at stalled replication forks (Bermudez-Lopez et al. 2010; Branzei et al. 2006). The Smc5/6 complex functions with Sgs1, a homolog of the Bloom syndrome helicase (BLM), to inhibit the accumulation of these abnormal intermediates. It is possible that this function is conserved in humans, as hypomorphic mutations that lead to the loss of the NSMCE2 SP-RING domain result in delayed recovery from replication stress and a reduction in BLM foci (Payne et al. 2014). These defects result in chromosome bridges and missegregation during the metaphase to anaphase transition. In budding yeast, $\mathrm{Smc} 5 / 6$ has been shown to interact and restrain the replication regression activity of Mph1 helicase, an ortholog of human FANCM, which is required for replication fork repair but can also lead to accumulation of JMs (Xue et al. 2014). 
In fission yeast, similar JMs accumulate at the collapsed replication forks in $s m c 6$ mutants, correlating with chromosome missegregation (Ampatzidou et al. 2006). Smc5/6 is required for the loading of Rpa and Rad52 onto stalled replication forks in order for the fork to maintain a recombination-competent conformation (Irmisch et al. 2009). Overexpression of Brc1, a BRCA C-terminal (BRCT) motif protein, rescues the replication-arresting defect of a Smc6 hypomorphic mutant (Lee et al. 2007; Sheedy et al. 2005; Verkade et al. 1999). Because this rescue is dependent on Brc1-mediated promotion of a post-replicative repair pathway and the function of structure-specific endonucleases Slx1/4 and Mus81/Eme1 that resolve the accumulated JMs, Smc5/6 complex may be required to prevent the formation of replication stressinduced $\mathrm{JMs}$ and/or assist in their resolution.

\section{Facilitating homologous recombination}

Numerous studies using mammalian, plant, budding yeast, and fission yeast cells have indicated that $\mathrm{Smc5} / 6$ functions in the homologous recombination pathway (Ampatzidou et al. 2006; Cost and Cozzarelli 2006; Lehmann et al. 1995; McDonald et al. 2003; Mengiste et al. 1999; Pebernard et al. 2006; Stephan et al. 2011; Torres-Rosell et al. 2005a, b; Watanabe et al. 2009).

In budding yeast and human cells, Smc5/6 and cohesin are recruited to DSBs to promote repair via sister chromatid recombination (De Piccoli et al. 2006; Lindroos et al. 2006; Potts et al. 2006; Strom et al. 2004; Unal et al. 2004; $\mathrm{Wu}$ and $\mathrm{Yu}$ 2012). Although Smc5/6 and cohesin complexes are recruited to DSBs independently, Nse2mediated sumoylation of the $\alpha$-kleisin subunit of cohesin, $\mathrm{Scc} 1$, is required to ensure proficient sister chromatid recombination (McAleenan et al. 2012; Wu and Yu 2012). In turn, sumoylation of Scc1 was shown to counteract the action of Wapl, a negative regulator of cohesin loading (Wu and Yu 2012).

ChIP experiments in mouse B cells showed that SMC5 co-localizes with RPA, the single-strand binding protein involved in DNA replication and repair, and BRCA1, a protein involved in DSB repair, at early replication fragile sites (Barlow et al. 2013). These findings suggest that the SMC5/6 complex binds to single-stranded DNA (ssDNA) substrates created during HR and/or DNA replication.

\section{Regulation of homologous recombination in repetitive sequences}

In budding yeast, the ribosomal genes are organized into a single array of 100-200 identical repeats on chromosome XII that is compartmentalized into the chromatin region called nucleolus (Oakes et al. 2006). Due to the repetitive nature of the ribosomal DNA (rDNA) locus, HR-mediated DNA damage repair in this region can lead to illegitimate recombination events that result in JMs and unequal sister chromatid exchange (Eckert-Boulet and Lisby 2009). In order to prevent such deleterious recombination events, DSBs occurring within rDNA are thought to be moved outside the nucleolus by a Smc5/6-dependent mechanism in order to be repaired (Torres-Rosell et al. 2005a, 2007). However, the visible presence of DSBs in the nucleolus of Smc5/6 mutants could also be due to less efficient repair of these breaks without functional Smc5/6.

Similarly, in Drosophila, Smc5/6 is thought to be involved in the translocation of the damaged DNA within heterochromatin regions to adjacent euchromatic regions where recombination can occur proficiently (Chiolo et al. 2011). Moreover, in heterochromatin, Smc5/6 suppresses HR until translocation of the DSB has occurred (Chiolo et al. 2011).

\section{Mitotic metaphase}

Smc6 location in mitotic metaphase cells has been studied multiple times, with varying outcomes. Some studies in mouse and human show that SMC6 is translocated away from the chromosomes during mitotic divisions (Gallego-Paez et al. 2014; Taylor et al. 2001; Verver et al. 2013, 2014), while other studies in budding yeast and mouse report Smc6 to be located at the centromeres of mitotic cells (Gomez et al. 2013; Lindroos et al. 2006; Yong-Gonzales et al. 2012).

The SMC5/6 complex is required for regulating topoisomerase II $\alpha$ and condensin localization on replicated chromatids in human cells during mitosis, thereby ensuring correct chromosome morphology and segregation (Gallego-Paez et al. 2014). Topoisomerase II (TopoII) resolves DNA topological constraints by introducing transient DSBs that are needed to decatenate double-stranded DNA to alleviate supercoiling (Nitiss 2009). TopoII initiates the passage of an unbroken DNA strand through the DSB and then reseals the break (Nitiss 2009). In budding yeast, Smc5/6 has recently been implicated in managing replication-induced topological stress (Carter and Sjogren 2012; Jeppsson et al. 2014a) and induction of topological stress by TopoII inactivation correlates with increased frequency of Smc5/6 chromosomal association sites (Jeppsson et al. 2014a; Kegel et al. 2011). In fission yeast, TopoII and Smc5/6 are required for the timely removal of cohesins from the chromosome arms before metaphase (Tapia-Alveal et al. 2010). Retention of these cohesins would otherwise cause chromosome missegregation and subsequent mitotic catastrophe. This was further supported when overexpression of separase, a protein that cleaves cohesin, was shown to rescue the lethality of TopoII and Smc5/6 mutants in fission yeast (Outwin et al. 2009). 


\section{Meiosis}

Meiosis is a specialized cell division during which one round of DNA replication is followed by two successive rounds of chromosome segregation. First, the homologous chromosomes, each consisting of one pair of sister chromatids held together by cohesin complexes, move to opposite poles (meiosis I). Second, the sister chromatids are segregated, resulting in the formation of four haploid cells (meiosis II). During prophase I, the homologous chromosomes align and, in most organisms, chromosome synapsis is achieved by formation of the synaptonemal complex (SC). Correct synapsis of the homologous chromosomes is required to facilitate meiotic recombination and the subsequent formation of meiotic crossovers. These meiotic crossovers, or chiasmata, introduce genetic variation among the resulting gametes. Additionally, together with proper sister chromatid cohesion, they also ensure correct chromosome orientation and segregation during meiosis I (reviewed in (Petronczki et al. 2003)).

The molecular pathways required for DSB repair during meiosis have been studied in most detail in budding yeast (De Muyt et al. 2012; Zakharyevich et al. 2012). However, evidence indicates that these pathways are conserved (Berchowitz et al. 2007; Higgins et al. 2008; Holloway et al. 2008). The following paragraphs briefly summarize meiotic recombination, using budding yeast as an example (Fig. 2). Meiotic recombination is initiated by Spo11-induced DSB formation, a 5-3' exonuclease that produces a 3' single-stranded DNA overhang at every break (Keeney et al. 1997). This 3' overhang is then coated by the Rad51/Dmc1 strand exchange proteins and invades the complementary sequence of the homologous chromosome (Fig. 2a). DNA synthesis then starts from the invading end and proceeds beyond the DSB. This single-end invasion (SEI) is the precursor of all recombination pathways during meiosis (De Muyt et al. 2012; Zakharyevich et al. 2012).

Following SEI, most recombination events are processed via synthesis-dependent single-strand annealing (SDSA) (Fig. 2b). During SDSA, the invading strand is thought to be displaced by the RecQ helicase BLM/Sgs1 (Bennett et al. 1998; De Muyt et al. 2012; Jessop and Lichten 2008; Jessop et al. 2006; Oh et al. 2008). The displaced strand is then used as a synthesis template for the other damaged ssDNA end, and ligation results in the formation of a non-crossover.

The DSB repair mechanism in budding yeast that ensures reciprocal crossover formation is known as the ZMM (Zip1-4, Mlh1/3, Msh4/5) pathway. The ZMM pathway requires both SC components (Zip1-4 and Spo16) and the conserved mismatch repair heterodimers MutS $\gamma$ (Msh4-5) and MutL $\gamma$ (Mlh1-3) (Borner et al. 2004; Lynn et al. 2007). At a ZMM designated recombination site, the SEI is stabilized and the second end of the DSB is captured to form a double Holliday junction (dHJ). Interestingly, Sgs1 is required to stabilize the ZMM designated $\mathrm{dHJ}$, which are resolved asymmetrically by Exo1-MutL $\gamma$ to form COs, and eventually lead to chiasmata (Zakharyevich et al. 2012) (Fig. 2c).

Timely organization of the different steps of meiotic DSB repair depends on tight regulation of the meiotic prophase I, which can be subdivided in four stages: leptonema, zygonema, pachynema, and diplonema. During leptonema, the chromatin condenses and formation of axial elements between sister chromatids begin to form. Simultaneously, DSBs are induced by the endonuclease SPO11, triggering the meiotic DNA damage response. During zygonema, homologous chromosomes begin to synapse, characterized by the formation of the SC, a proteinaceous structure which comprises axial proteins (now termed lateral elements) linked by central components. Single-strand invasion occurs, followed by resection and DNA synthesis, resulting in recombination intermediates. Recombination events are neither randomly nor equally distributed throughout the genome but are preferentially located at hotspots at which DSBs are more frequently formed (reviewed in (Keeney et al. 2014)). At pachynema, the homologous chromosomes are fully synapsed along their entire length. DSB repair via HR continues by the resolution of recombination intermediates into either a non-crossover or a crossover event. Only a minority of recombination intermediates are resolved as crossovers, but there are processes which ensure that at least one crossover is formed per homolog pair (reviewed in (Youds and Boulton 2011)). Finally, in diplonema, the synaptonemal complex gradually dissociates and most recombination intermediates are completely resolved. Importantly, crossovers remain as chiasmata in order to keep homologous chromosomes locally tethered and, together with proper chromosome cohesion, ensure biorientation and accurate segregation during meiosis I (reviewed in (Petronczki et al. 2003)).

During the first meiotic division, homologous chromosomes, each containing two sister chromatids held together by cohesins, segregate to opposite poles. Bi-orientation of homologous chromosomes is crucial for their accurate segregation, and misalignment may result in aneuploidy. The spindle assembly checkpoint (SAC) controls this bi-orientation by monitoring the tension that is generated when the homologous chromosomes are pulled to opposite directions and only allows subsequent chromosome segregation when all chromosomes are correctly orientated. The physical linkage that chiasmata provide achieves bi-orientation and inter-homolog tension. Failure to generate the chiasmata, e.g., due to absence of DSB induction, inadequate repair, and lack of $\mathrm{CO}$ events, will lead to either a SAC induced metaphase I arrest and apoptosis or aberrant chromosome segregation and aneuploidy in the resulting gametes. 


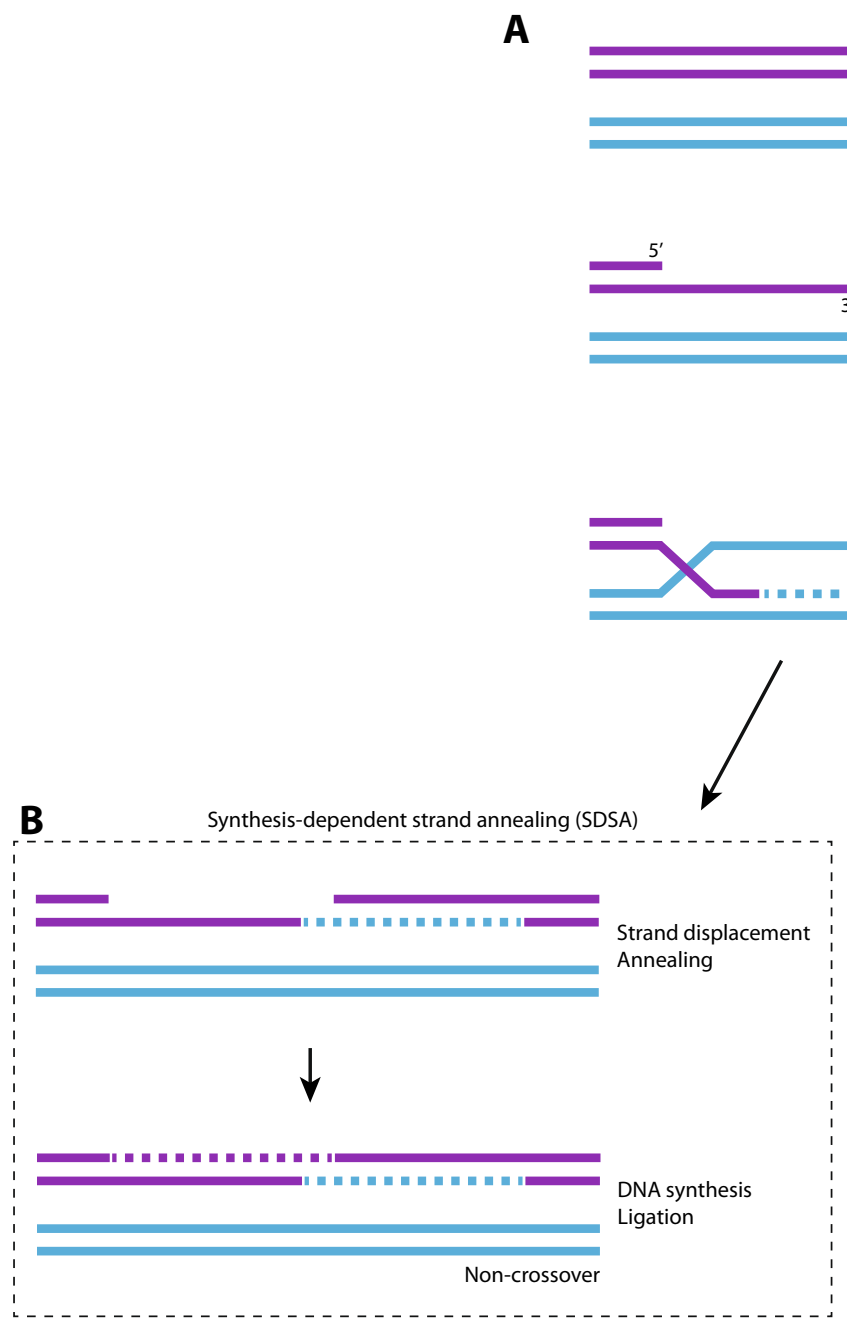

Fig. 2 DNA double strand break repair by homologous recombination. a When a DNA double strand break (DSB) occurs, the DNA around the 5' end is resected, creating a $3^{\prime}$ single-stranded DNA (ssDNA) overhang. This $3^{\prime}$ ssDNA overhang invades a homologous sequence, creating a Dloop. DNA is synthesized at the invading end using the undamaged template DNA strand. After this, further repair can be executed by synthesis-dependent strand annealing (SDSA) or double strand break repair (DSBR). b SDSA: The second DSB end will be annealed up to

\section{Localization of Smc5/6 in meiosis}

\section{Budding yeast}

Using immunofluorescence microscopy, Smc6 was observed to localize to the nucleolus in budding yeast at the entry into meiosis (Farmer et al. 2011; Lilienthal et al. 2013). During meiotic progression, chromosome axes are formed and DSB repair is initiated. At this time, Smc5 and Smc6 localize as distinct foci along the chromosome axes (Copsey et al. 2013;

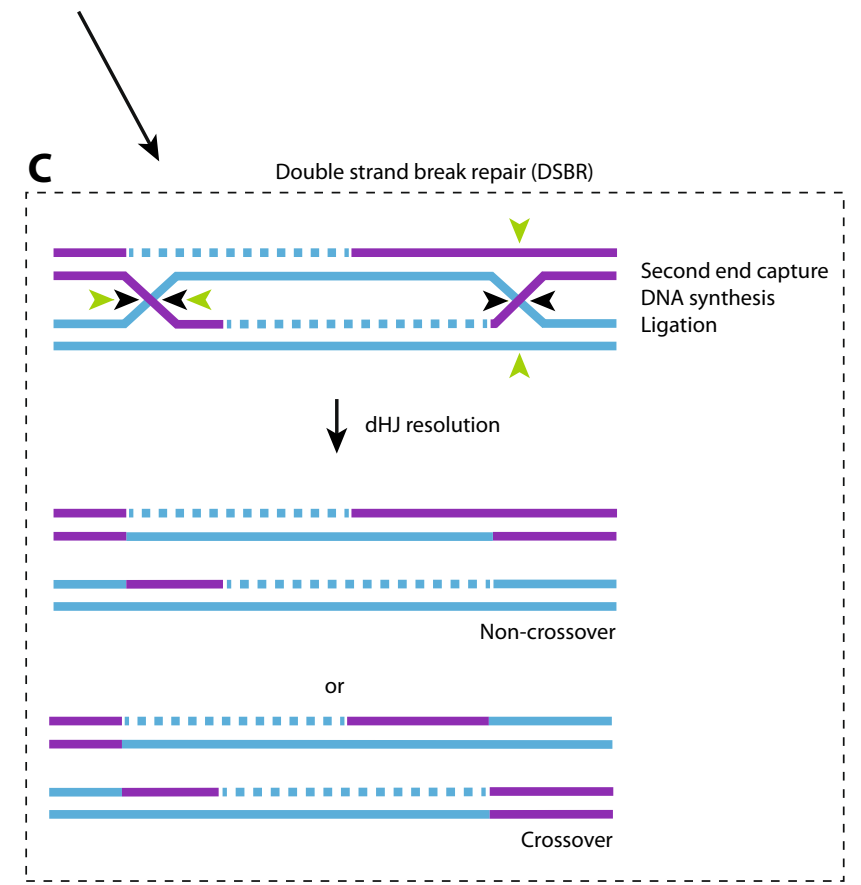

the ssDNA on the other break end, followed by gap-filling DNA synthesis and ligation. This will lead to a non-crossover event. c DSBR: The second DSB end can be captured to form a double Holliday Junction (HJ). The resulting recombination intermediate must be resolved by nicking the HJs. Depending on the nick sites, either parallel (black arrows) or anti-parallel (green arrows), this will produce a noncrossover or a crossover event, respectively

Farmer et al. 2011; Lilienthal et al. 2013; Xaver et al. 2013). Smc6 also frequently co-localizes side by side with Rad51 recombinase, indicating a potential function in the strand invasion step in HR repair (Copsey et al. 2013; Xaver et al. 2013). The Smc6 localization along the axes becomes more abundant as synapsis occurs (Copsey et al. 2013; Lilienthal et al. 2013; Xaver et al. 2013). The formation of this punctate distribution does not depend on meiotic DSBs (Copsey et al. 2013; Farmer et al. 2011). Contrasting data has been reported for the effect of cohesin mutation on Smc5/6 axis loading. It 
was observed by Lillienthal et al. that Smc6 binding to chromosomes is dependent on meiosis-specific cohesin subunit Rec8. Therefore, the Smc5/6 complex may be influenced by meiotic axis structure and/or the presence of sister chromatid cohesion. In contrast, however, the localization of Smc5 was not affected by the absence of Rec8 (Copsey et al. 2013). Although surprising, it is possible that Smc5 and Smc6 loading to chromosome axes is independent of one another, and Smc6 but not Smc5 requires cohesin. An alternative explanation is that differences in chromatin spreading techniques resulted in the contrasting observations. The localization of Smc5/6 during late prophase is still inconclusive. After late prophase, some studies reported that Smc5 and Smc6 localization become more diffuse and are absent prior to metaphase I (Copsey et al. 2013; Lilienthal et al. 2013), while another study reported that Smc6 localized to the chromatin during both meiotic divisions, displaying dense clusters at the boundary between segregating chromatin masses (Xaver et al. 2013). These discrepancies may be due to sensitivity differences in chromatin spreading technique and epitope accessibility.

To assess chromatin localization of Smc5/6 in greater detail, genome-wide ChIP-on-chip localization studies were used (Copsey et al. 2013; Xaver et al. 2013). These studies showed that Smc5 and Smc6 bind to many of the same chromosomal axis-associated sites as Rec8, including centromeres. In addition, Smc5/6 is enriched at DSB hotspots. However, this localization occurs independently of DSB formation, which supports the immunofluorescence microscopy data (Copsey et al. 2013; Xaver et al. 2013). Finally, as observed in mitotic cells, Smc5/6 also binds to the rDNA, which remains unsynapsed during meiotic prophase I (Farmer et al. 2011; Lilienthal et al. 2013; Xaver et al. 2013).

\section{Caenorhabditis elegans}

In C. elegans, SMC-6 localizes to the condensed chromatin of germ cells throughout meiosis (Bickel et al. 2010). SMC-6 becomes enriched on chromosomes during pachytene, which coincides with occurrence of DSB repair, complementing the localization pattern in budding yeast. SMC-6 remains on chromosome axes during diplotene and diakinesis in worms (Bickel et al. 2010).

\section{Mouse, human}

The first indications of a possible role for SMC5/6 in mammalian meiotic progression were elevated levels of both SMC5 and SMC6 in the testis and localization in spermatocytes (Taylor et al. 2001). It then took over 12 years before the role of SMC5/6 in mammalian meiosis was investigated in more depth revealing involvement at several crucial and diverse steps during rodent and human spermatogenesis
(Gomez et al. 2013; Verver et al. 2013, 2014). First, in mouse spermatocytes, SMC5, SMC6, and NSMCE1 were found to be located at pericentromeric heterochromatin (or so-called chromocenters): condensed repetitive sequences surrounding the centromeres (Gomez et al. 2013; Verver et al. 2013). This localization already starts in differentiating spermatogonia, remains throughout all meiotic stages, including metaphase I and II, and disappears when the haploid spermatids start to elongate (Gomez et al. 2013; Verver et al. 2013). Moreover, SMC5 and SMC6 were detected at the SC of synapsed homologous chromosomes from early zygonema until late diplonema in mouse spermatocytes (Gomez et al. 2013). This latter localization pattern was also reported for both SMC5 and SMC6 in human spermatocytes (Verver et al. 2014). Finally, detection of SMC5, SMC6, and NSMCE1 at the XY body during pachynema was observed in both mouse (Gomez et al. 2013; Taylor et al. 2001) and human spermatocytes (Verver et al. 2014). However, it must be noted that in mouse spermatocytes, SMC5, SMC6, and NSMCE1 localize to the chromatin of the XY body (Gomez et al. 2013), whereas in human spermatocytes, the localization of SMC6 was limited to distinct foci located at the axial elements of the unsynapsed $X$ and $Y$ chromosomes (Verver et al. 2014).

\section{Functions of Smc5/6 in meiosis}

\section{Meiotic recombination}

When meiotic recombination intermediates are not properly resolved to form either a non-crossover or crossover, aberrant joint molecules (JMs) can emerge. These JMs have the potential to block chromosome segregation if unresolved (Copsey et al. 2013; Jessop and Lichten 2008; Xaver et al. 2013). Sgs1 limits the formation of these JM structures (Chen et al. 2010; De Muyt et al. 2012; Fabre et al. 2002; Jessop and Lichten 2008; Sugawara et al. 2004). Several structure-selective nucleases, Mus81-Mms4, Slx1-Slx4, and Yen1, are involved in the resolution in these JMs (De Muyt et al. 2012; Matos et al. 2011; Zakharyevich et al. 2012). In budding yeast, the Smc5/6 complex antagonizes the formation of JMs via two mechanisms: (i) prevention of JMs by destabilizing SEI intermediates (Xaver et al. 2013) and (ii) facilitating JM resolution (Copsey et al. 2013; Lilienthal et al. 2013; Xaver et al. 2013). Like previously reported for the helicase BLM/Sgs1, the SUMO E3 ligase function of Nse2/Mms21 subunit is required to destabilize SEI intermediates (Xaver et al. 2013). This inhibition is needed to prevent the formation of inappropriate recombination intermediates. In the absence of Smc5/6, these inappropriate recombination intermediates develop into JMs that require the structure-selective resolvases Mus81-Mms4, Slx1- 
Slx4, and Yen 1 to be processed (Zakharyevich et al. 2012). Of these resolvases, at least the ability of Mus 81 to associate with, or be stabilized on, the meiotic chromosomes efficiently is dependent on Smc5/6 (Copsey et al. 2013). Interestingly, while required to limit SEI stabilization, the SUMO E3 ligase function of Nse2/Mms21 is not required for Smc5/6 directed JM resolution (Xaver et al. 2013).

In fission yeast, meiotic recombination generates single Holliday junction (HJ) intermediates (Cromie et al. 2006; Davis and Smith 2003; Hyppa and Smith 2010; Keeney et al. 1997), which are eventually resolved by the Mus81Eme1 complex (Boddy et al. 2001; Cromie et al. 2006; Osman et al. 2003). Based on genetic experiments, the Smc5/6 complex subunits Nse5-Nse6 have a regulatory role in Mus81-Eme1 dependent HJ resolution (Wehrkamp-Richter et al. 2012).

In C. elegans, the SMC-5/6 complex is not required for chiasmata formation. However, mutation of $s m c-5$ or $s m c-6$ did result in chromosome fragmentation during meiosis I and an increased number of RAD-51 foci in the nucleus (Bickel et al. 2010). Interestingly, mus-81, him-6 (a BLM ortholog), and $m u s-81, x p f-1$ double mutants display a similar phenotype to the $s m c-5$ or $s m c-6$ mutants (O'Neil et al. 2013). Because these genes are involved in two redundant $\mathrm{HJ}$ resolution pathways in C. elegans (Agostinho et al. 2013), the SMC-5/6 complex is likely to be involved in $\mathrm{HJ}$ resolution. Hence, the C. elegans SMC-5/6 complex may be playing similar JM antagonistic roles observed in budding yeast by hindering $\mathrm{JM}$ formation early and assisting JM resolution. However, C. elegans chromosomes are holocentric, and subsequent roles of SMC-5/6 in chromosome segregation may differ from other model organisms.

\section{Preventing HR in heterochromatin}

In budding yeast, Smc5/6 also binds to the rDNA, which remains unsynapsed during meiotic prophase I (Farmer et al. 2011; Lilienthal et al. 2013; Xaver et al. 2013). Smc5/6 has been shown to have an anti-recombinogenic role at this repetitive DNA locus during vegetative growth (Torres-Rosell et al. 2007). Additionally, budding yeast Smc6 is strongly enriched in the pericentromeric regions during the mitotic G2 phase (Lindroos et al. 2006). Smc5/6 is essential for the timely separation of chromatids and the prevention of branched and entangled chromosome structures and subsequent mitotic arrest (Lindroos et al. 2006). It is conceivable that $\mathrm{Smc} 5 / 6$ plays similar roles at the rDNA locus and pericentromeric regions during meiosis.

In mouse spermatocytes, SMC5, SMC6, and NSMCE1 localize at pericentromeric heterochromatin (Gomez et al. 2013; Verver et al. 2013). As with rDNA, these regions are at high risk of aberrant recombination events when HR is enabled, leading to genomic instability (Goodarzi and Jeggo 2012). An additional challenge specific to meiotic cells is the endogenous induction of DSBs that are repaired by HR. Pericentromeric heterochromatin consists of densely packed repetitive sequences and is therefore vulnerable to aberrant events such as the formation of intra-chromosomal recombination structures. As a result, meiotic recombination is generally suppressed around the centromeres (Lynn et al. 2004), via a mechanism yet to be elucidated. The role of Smc6 in preventing HR in these high-risk regions has already been established for yeast and Drosophila mitotic cells (Chiolo et al. 2011; Torres-Rosell et al. 2007). In line with these studies, pericentromeric heterochromatin of mouse prophase spermatocytes is simultaneously marked with SMC5, SMC6, and NSMCE1 (Gomez et al. 2013; Verver et al. 2013) and deprived of recombination sites marked by RAD51 (Verver et al. 2013). These findings suggest that also in mammalian germ cells, SMC5/6 might be responsible for preventing aberrant HR events in repetitive sequences. Interestingly, even though prevention of HR in heterochromatin might be a conserved function of SMC5/6, a similar localization was not found in human prophase spermatocytes (Verver et al. 2014).

\section{Centromere cohesion}

During budding yeast meiosis, Smc5/6 regulates sister chromatid cohesion at centromeres and is required for the timely removal of cohesin from chromosomal arms (Copsey et al. 2013).

SMC6 is proximal to the centromeres during both meiotic metaphases in mouse (Gomez et al. 2013; Verver et al. 2013) and human (Verver et al. 2014). As well as during prophase I stages, SMC6 co-localizes at the centromeres with Topo II $\alpha$ during metaphase I and II (Gomez et al. 2013). More specifically, in metaphase I and anaphase I, SMC6 was present as two foci proximal to the sister kinetochores, and only one signal near the kinetochores at metaphase II and anaphase II (Gomez et al. 2013). Additionally, in metaphase II spermatocytes, in which the centromeres are subjected to tension from opposite poles, SMC6 appeared as a strand connecting the sister kinetochores (Gomez et al. 2013). The finding that SMC6 co-localizes with Topo II $\alpha$, together with the fact that the strand of SMC6 joining sister kinetochores persists even after redistribution of Aurora-B, suggests that the SMC5/6 complex may regulate sister chromatid centromere cohesion and dissolution of DNA catenates that form after DNA replication (Gomez et al. 2013). This role for SMC5/6 was further appointed when Topo II $\alpha$ was inhibited by etoposide, inducing lagging chromosomes during the second meiotic division. Both SMC6 and Topo II $\alpha$ co-localized at stretched strands connecting these lagging chromatids at the site of the kinetochores (Gomez et al. 2013). Complementary data was acquired using budding yeast, where localization of Smc5 
depends on meiotic DNA replication, and in the absence of TopoII, Smc5 localization is aberrant (Copsey et al. 2013).

\section{SC assembly/stability, homologous chromosome synapsis}

Both in mouse and human spermatocytes, SMC5 and SMC6 were found to be located at the SC (Gomez et al. 2013; Verver et al. 2014). Co-localization of mouse SMC6 with the SC central region proteins SYCP1 and TEX12 showed that SMC6 is restricted to synapsed chromosomes, leaving the un- or desynapsed axes including $\mathrm{X}$ and $\mathrm{Y}$, unmarked (Gomez et al. 2013). Mammalian synapsis is characterized by the presence of a central region that, besides SYCP1 (equivalent to Zip1 in budding yeast), also contains the central element proteins SYCE1-3 and TEX12 (Bolcun-Filas et al. 2007, 2009; Hamer et al. 2006, 2008; Schramm et al. 2011). However, although dependent on SYCP1, loading of SMC6 to the mouse SC occurs independent of these central element proteins (Gomez et al. 2013). Additionally, mouse SMC5/6 localization is not dependent on meiosis-specific cohesin subunits REC8 and SMC1 $\beta$ (Gomez et al. 2013). The longitudinal localization pattern along the mammalian synapsed SC axes could suggest that localization of SMC5/6 is dictated by chromosome structure, as has been suggested in mitotic cells (Jeppsson et al. 2014b), or that the complex either facilitates SC assembly, chromosome synapsis, or recruitment of other SC-associated proteins.

\section{The XY body and unsynapsed chromosomes in pachytene spermatocytes}

In males, due to a lack of homology, the $\mathrm{X}$ and $\mathrm{Y}$ chromosomes remain largely unsynapsed during the meiotic prophase I. During meiotic prophase, unsynapsed chromosomal regions are transcriptionally silenced by a process called meiotic silencing of unsynapsed chromosomes (MSUC) (Ichijima et al. 2012). In the case of the $X$ and $Y$ chromosome, this silencing is called meiotic sex chromosome inactivation (MSCI), and is achieved by the formation of a so-called XY body (or sex body), marked by the presence of several DNA damage response proteins such as BRCA1, $\gamma$-H2AX, and ATR (Ichijima et al. 2012). In male meiotic cells with extensive autosomal asynapsis, MSUC competes with MSCI for these proteins. The sex chromosomes will then be inadequately silenced, which will result in a pachytene arrest (Burgoyne et al. 2009). In mouse spermatocytes, SMC5, SMC6, and NSMC E1 were found to cover the XY body (Gomez et al. 2013). Because the XY staining resembles that of $\gamma-\mathrm{H} 2 \mathrm{AX}$, it is proposed that the SMC5/6 complex might be facilitating MSCI at this site.

In human spermatocytes, SMC6 is present on the unsynapsed $\mathrm{XY}$ chromosomes in a more foci-like pattern (Verver et al. 2014), suggesting a function in DSB repair.
Interestingly, it has been recently found that in the absence of synapsis, including the unsynapsed regions of the sex chromosomes, SPO11 will continue to make DSBs (Kauppi et al. 2013). In this light, the presence of SMC5/6 on the unsynapsed sex chromosomes might be required to repair these continuously induced DSBs. In addition to this observation, unsynapsed autosomes display both RAD51 and SMC6 foci (Verver et al. 2014). Hence, it seems likely that human SMC5/ 6 plays a role in the repair of the continuously induced DSBs on unsynapsed meiotic chromosomes.

\section{Discussion/concluding remarks}

In recent years, assessment of Smc5/6 localization and analysis of Smc5/6 mutant phenotypes during meiosis has resulted in an abundance of data implying a number of meiotic functions (Table 1). In all models, and in line with its described functions during mitosis, Smc5/6 is involved in HR-mediated repair and chromosome segregation, as depicted in Fig. 3. However, despite this common denominator, the meiotic functions of Smc5/6 seem astonishingly diverse.

Several studies in mammalian models have shown varying results when using antibodies against different epitopes of SMC6 simultaneously (Gomez et al. 2013; Verver et al. 2013, 2014). Since the technical variation within experiments was negligible, differences in localization pattern are most likely a reflection of varying conformations of the SMC6 protein or SMC5/6 complex as a whole, resulting in differing accessibility of these epitopes. Indeed, a study using budding yeast demonstrates that the Smc5/6 complex is physically remodeled in an ATP-dependent manner (Bermudez-Lopez et al. 2015). Even though future studies might unravel the role of conformation herein, another possibility is that Smc6 and/ or Smc5 can act independently from the Smc5/6 complex, thereby showing differential localization patterns. When budding yeast proteins were purified separately, Smc5 and Smc6 were found to have some binding activity to ssDNA, independently of the presence of the other subunits (Roy and D'Amours 2011; Roy et al. 2011). However, even though some studies support the complex-independent function of Smc5 and Smc6 (Laflamme et al. 2014; Roy et al. 2011; Vignard et al. 2011), most studies show that hypomorphic alleles and RNAi knockdown of Smc5 and Smc6 yield complementary phenotypes (e.g., (Gallego-Paez et al. 2014; Torres-Rosell et al. 2005b)). Moreover, fractionation experiments indicate that the majority of Smc5/6 components are in complex, and only a small fraction is present as isolated monomers (Torres-Rosell and Losada 2011).

The diversity of mitotic and meiotic functions of Smc5/6 illustrates the versatility of this protein complex. Yet, the regions where Smc5/6 has been found to act are not random and its roles on rDNA, telomeres, DSBs, replication sites, and 


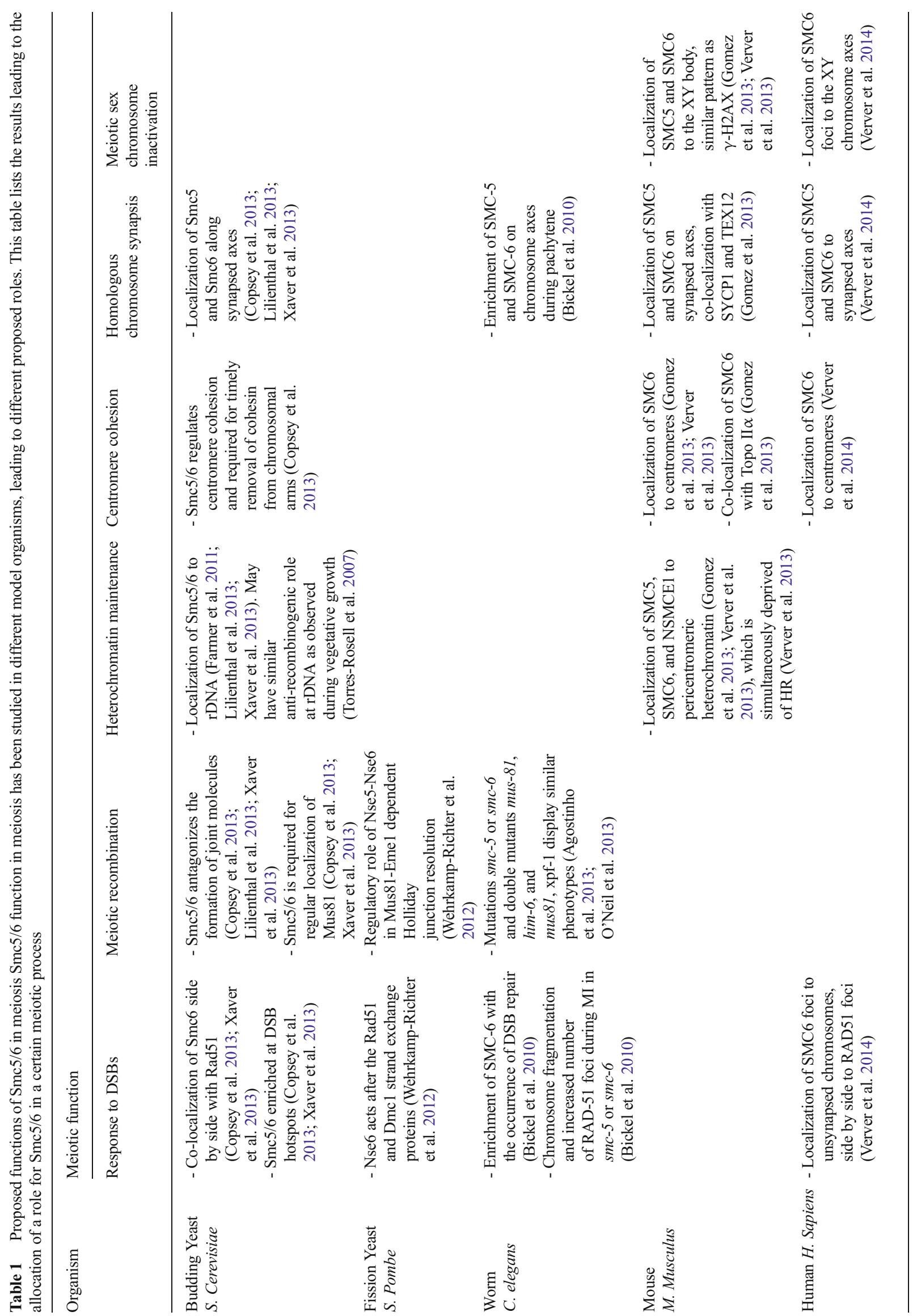


Fig. 3 Proposed functions of Smc5/6 in meiosis. a In budding and fission yeast, Smc5/6 is required for the resolution of meiotically induced joint molecules and correct segregation of homologous chromosomes. Without functional Smc5/6 recombination intermediates cannot be efficiently resolved, leading to the accumulation of inter-homolog, inter-sister, and multi-chromatid joint molecules and failure to segregate chromosomes properly. Black $s p o t=$ centromere. $\mathbf{b}$ During mouse and human meiosis, SMC5/6 functions in a variety of processes. It is proposed to be involved in synaptonemal complex formation and/or stability, heterochromatin maintenance, and XY body silencing. Moreover, it may be required for repair of DSBs due to lack of synapsis and resolving meiotic recombination intermediates. Finally, SMC5/6 is involved in centromere cohesion during M-phase. Purple=SMC5/ 6 complex localization. Gray filaments =lateral elements of the synaptonemal complex. Gray spot $=$ centromere. Note: depicted chromosomes represent (telocentric) mouse chromosomes
A

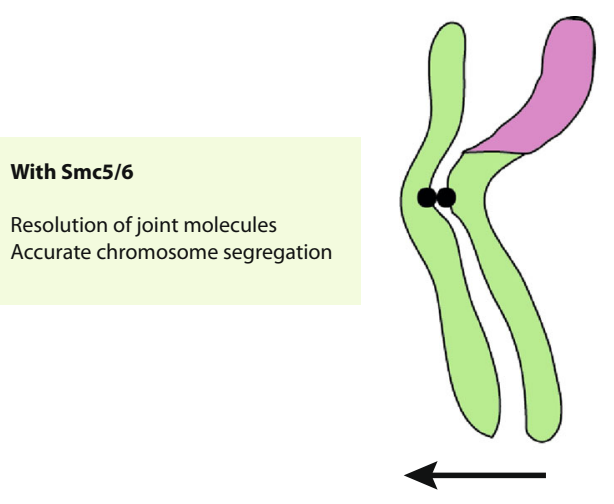

\section{Without Smc5/6}

Accumulation and inability to resolve joint molecules including Inter-homolog (IH), Inter-sister (IS) and multi-chromatid joint molecules

Failure to segregate chromosomes

B

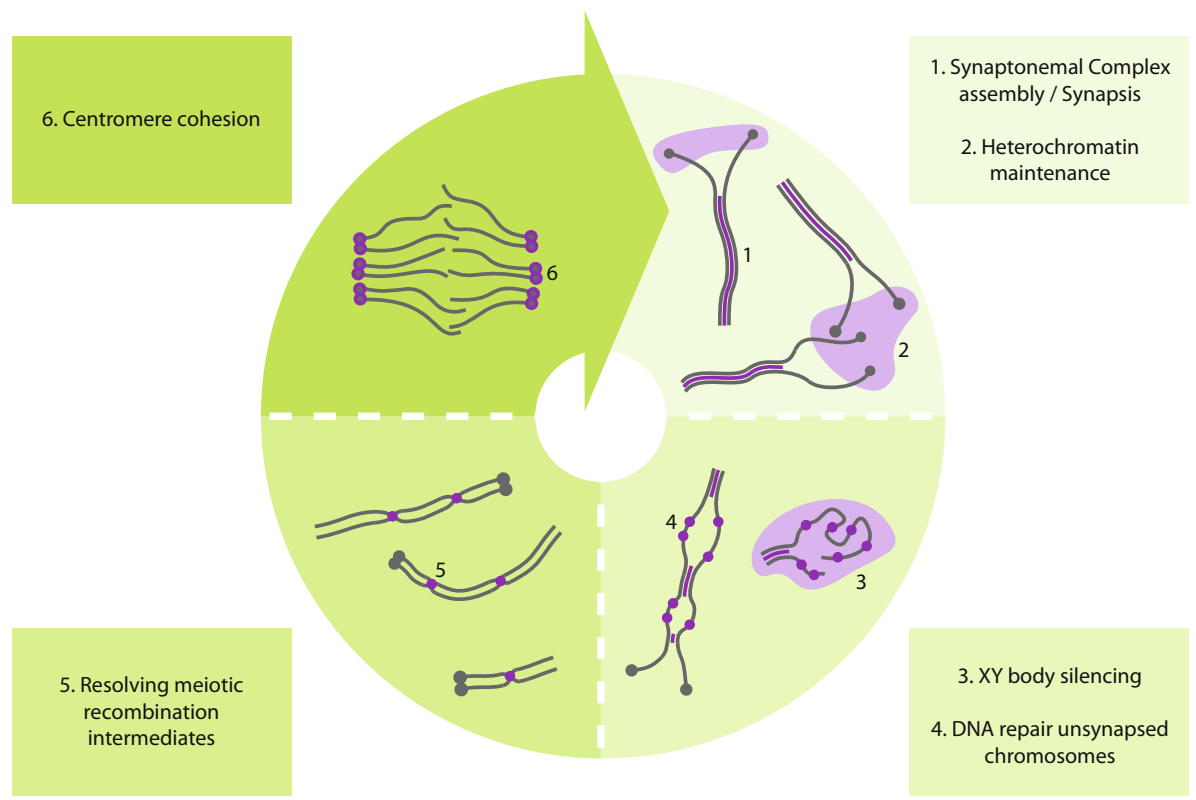

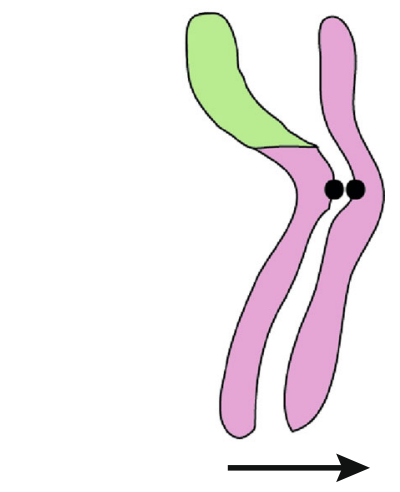

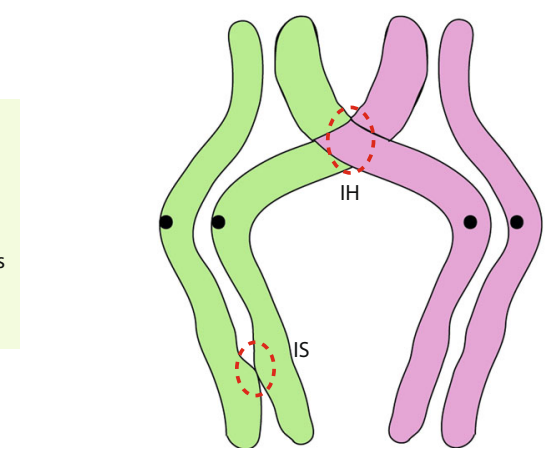

Meiosis I

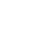

collapsed replication forks all support the strong preference of Smc5 and Smc6 to capture ssDNA (Roy and D'Amours 2011; Roy et al. 2011). The consequences of Smc5/6 binding to DNA seem to vary between the specific processes it is required for. During meiosis, the Smc5/6 complex can either 
seemingly diverse roles during different processes, resolving complex chromosome structures, which would otherwise cause cell cycle arrest or prevent chromosomes from being segregated, appears a major meiotic function of Smc5/6. However, how Smc5/6 is molecularly regulated during different meiotic processes, such as pre-meiotic S-phase, meiotic recombination, and the meiotic M-phases, still needs further research. Creation and assessment of mammalian mutant models, together with the development of a comprehensive meiosis interactome for the SMC5/6 complex will further our comprehension of SMC5/6 functions. More knowledge on the meiotic functions of Smc5/6 may give insight in one of the biggest questions in biology: how are germ cells capable to passage their genome through essentially endless generations while maintaining sufficient genomic integrity.

Acknowledgments This work is supported by an AMC Fellowship and The People Programme (Marie Curie Actions) of the European Union's Seventh Framework Programme (CIG 293765) to GH and a NIH R00 award HD069458 to PWJ.

\section{Compliance with ethical standards}

Funding This work is supported by an AMC Fellowship and The People Programme (Marie Curie Actions) of the European Union's Seventh Framework Programme (CIG 293765) to GH and a NIH R00 award HD069458 to PWJ.

Conflict of interest The authors declare that they have no conflict of interest. This article does not contain any studies with human participants or animals performed by any of the authors.

Open Access This article is distributed under the terms of the Creative Commons Attribution 4.0 International License (http:// creativecommons.org/licenses/by/4.0/), which permits unrestricted use, distribution, and reproduction in any medium, provided you give appropriate credit to the original author(s) and the source, provide a link to the Creative Commons license, and indicate if changes were made.

\section{References}

Agostinho A et al (2013) Combinatorial regulation of meiotic holliday junction resolution in C. elegans by HIM-6 (BLM) helicase, SLX-4, and the SLX-1, MUS-81 and XPF-1 nucleases. PLoS Genet 9, e1003591

Ampatzidou E, Irmisch A, O’Connell MJ, Murray JM (2006) Smc5/6 is required for repair at collapsed replication forks. Mol Cell Biol 26: 9387-9401

Andrews EA, Palecek J, Sergeant J, Taylor E, Lehmann AR, Watts FZ (2005) Nse2, a component of the Smc5-6 complex, is a SUMO ligase required for the response to DNA damage. Mol Cell Biol 25:185-196

Barlow JH et al (2013) Identification of early replicating fragile sites that contribute to genome instability. Cell 152:620-632

Bennett RJ, Sharp JA, Wang JC (1998) Purification and characterization of the Sgs1 DNA helicase activity of Saccharomyces cerevisiae. J Biol Chem 273:9644-9650
Berchowitz LE, Francis KE, Bey AL, Copenhaver GP (2007) The role of AtMUS81 in interference-insensitive crossovers in A. thaliana. PLoS Genet 3, e132

Bermudez-Lopez M, Ceschia A, de Piccoli G, Colomina N, Pasero P, Aragon L, Torres-Rosell J (2010) The Smc5/6 complex is required for dissolution of DNA-mediated sister chromatid linkages. Nucleic Acids Res 38:6502-6512

Bermudez-Lopez M et al (2015) ATPase-Dependent Control of the Mms21 SUMO Ligase during DNA Repair. PLoS Biol 13, e1002089

Bickel JS, Chen L, Hayward J, Yeap SL, Alkers AE, Chan RC (2010) Structural maintenance of chromosomes (SMC) proteins promote homolog-independent recombination repair in meiosis crucial for germ cell genomic stability. PLoS Genet 6, e1001028

Boddy MN, Gaillard PH, McDonald WH, Shanahan P, Yates JR 3rd, Russell P (2001) Mus81-Eme1 are essential components of a Holliday junction resolvase. Cell 107:537-548

Bolcun-Filas E, Costa Y, Speed R, Taggart M, Benavente R, De Rooij DG, Cooke HJ (2007) SYCE2 is required for synaptonemal complex assembly, double strand break repair, and homologous recombination. J Cell Biol 176:741-747

Bolcun-Filas E et al (2009) Mutation of the mouse Sycel gene disrupts synapsis and suggests a link between synaptonemal complex structural components and DNA repair. PLoS Genet 5, e1000393

Borner GV, Kleckner N, Hunter N (2004) Crossover/noncrossover differentiation, synaptonemal complex formation, and regulatory surveillance at the leptotene/zygotene transition of meiosis. Cell 117:29-45

Branzei D et al (2006) Ubc9- and mms21-mediated sumoylation counteracts recombinogenic events at damaged replication forks. Cell 127:509-522

Burgoyne PS, Mahadevaiah SK, Turner JM (2009) The consequences of asynapsis for mammalian meiosis. Nat Rev 10:207-216

Carter SD, Sjogren C (2012) The SMC complexes, DNA and chromosome topology: right or knot? Crit Rev Biochem Mol Biol 47:1-16

Chen C et al (2010) Meiosis-specific gene discovery in plants: RNA-Seq applied to isolated Arabidopsis male meiocytes. BMC Plant Biol 10: 280

Chiolo I, Minoda A, Colmenares SU, Polyzos A, Costes SV, Karpen GH (2011) Double-strand breaks in heterochromatin move outside of a dynamic HP1a domain to complete recombinational repair. Cell 144:732-744

Copsey A et al (2013) Smc5/6 coordinates formation and resolution of joint molecules with chromosome morphology to ensure meiotic divisions. PLoS Genet 9, e1004071

Cost GJ, Cozzarelli NR (2006) Smc5p promotes faithful chromosome transmission and DNA repair in Saccharomyces cerevisiae. Genetics 172:2185-2200

Cromie GA, Hyppa RW, Taylor AF, Zakharyevich K, Hunter N, Smith GR (2006) Single Holliday junctions are intermediates of meiotic recombination. Cell 127:1167-1178

Davis L, Smith GR (2003) Nonrandom homolog segregation at meiosis I in Schizosaccharomyces pombe mutants lacking recombination. Genetics 163:857-874

De Muyt A, Jessop L, Kolar E, Sourirajan A, Chen J, Dayani Y, Lichten M (2012) BLM helicase ortholog Sgs1 is a central regulator of meiotic recombination intermediate metabolism. Mol Cell 46:43-53

De Piccoli G et al (2006) Smc5-Smc6 mediate DNA double-strand-break repair by promoting sister-chromatid recombination. Nat Cell Biol 8:1032-1034

De Piccoli G, Torres-Rosell J, Aragon L (2009) The unnamed complex: what do we know about Smc5-Smc6? Chromosome Res 17:251263

Doyle JM, Gao J, Wang J, Yang M, Potts PR (2010) MAGE-RING protein complexes comprise a family of E3 ubiquitin ligases. Mol Cell 39:963-974 
Duan X, Yang Y, Chen YH, Arenz J, Rangi GK, Zhao X, Ye H (2009) Architecture of the Smc5/6 complex of saccharomyces cerevisiae reveals a unique interaction between the Nse5-6 subcomplex and the hinge regions of Smc5 and Smc6. J Biol Chem 284:8507-8515

Eckert-Boulet N, Lisby M (2009) Regulation of rDNA stability by sumoylation. DNA Repair 8:507-516

Fabre F, Chan A, Heyer WD, Gangloff S (2002) Alternate pathways involving Sgs1/Top3, Mus81/ Mms4, and Srs2 prevent formation of toxic recombination intermediates from single-stranded gaps created by DNA replication. Proc Natl Acad Sci U S A 99:1688716892

Farmer S, San-Segundo PA, Aragón L (2011) The smc5-smc6 complex is required to remove chromosome junctions in meiosis. PLoS One 6, e20948. doi:10.1371/journal.pone.0020948

Fujioka Y, Kimata Y, Nomaguchi K, Watanabe K, Kohno K (2002) Identification of a novel non-structural maintenance of chromosomes (SMC) component of the SMC5-SMC6 complex involved in DNA repair. J Biol Chem 277:21585-21591

Gallego-Paez LM et al (2014) Smc5/6-mediated regulation of replication progression contributes to chromosome assembly during mitosis in human cells. Mol Biol Cell 25:302-317

Gomez R et al (2013) Dynamic localization of SMC5/6 complex proteins during mammalian meiosis and mitosis suggests functions in distinct chromosome processes. J Cell Sci 126:4239-4252

Goodarzi AA, Jeggo PA (2012) The heterochromatic barrier to DNA double strand break repair: how to get the entry visa. Int J Mol Sci 13:11844-11860

Hamer G, Gell K, Kouznetsova A, Novak I, Benavente R, Höög C (2006) Characterization of a novel meiosis-specific protein within the central element of the synaptonemal complex. J Cell Sci 119:40254032. doi: $10.1242 /$ jcs. 03182

Hamer G, Novak I, Kouznetsova A, Hoög C (2008) Disruption of pairing and synapsis of chromosomes causes stage-specific apoptosis of male meiotic cells. Theriogenology 69:333-339. doi:10.1016/j. theriogenology.2007.09.029

Hazbun TR et al (2003) Assigning function to yeast proteins by integration of technologies. Mol Cell 12:1353-1365

Higgins JD, Buckling EF, Franklin FC, Jones GH (2008) Expression and functional analysis of AtMUS81 in Arabidopsis meiosis reveals a role in the second pathway of crossing-over. Plant J 54:152-162

Holloway JK, Booth J, Edelmann W, McGowan CH, Cohen PE (2008) MUS81 generates a subset of MLH1-MLH3-independent crossovers in mammalian meiosis. PLoS Genet 4, e1000186

Hyppa RW, Smith GR (2010) Crossover invariance determined by partner choice for meiotic DNA break repair. Cell 142:243-255

Ichijima Y, Sin HS, Namekawa SH (2012) Sex chromosome inactivation in germ cells: emerging roles of DNA damage response pathways. Cell Mol Life Sci 69:2559-2572

Irmisch A, Ampatzidou E, Mizuno K, O’Connell MJ, Murray JM (2009) Smc5/6 maintains stalled replication forks in a recombinationcompetent conformation. EMBO J 28:144-155

Jeppsson K et al (2014a) The chromosomal association of the Smc5/6 complex depends on cohesion and predicts the level of sister chromatid entanglement. PLoS Genet 10, e1004680

Jeppsson K, Kanno T, Shirahige K, Sjogren C (2014b) The maintenance of chromosome structure: positioning and functioning of SMC complexes. Nat Rev 15:601-614

Jessop L, Lichten M (2008) Mus81/Mms4 endonuclease and Sgs1 helicase collaborate to ensure proper recombination intermediate metabolism during meiosis. Mol Cell 31:313-323

Jessop L, Rockmill B, Roeder GS, Lichten M (2006) Meiotic chromosome synapsis-promoting proteins antagonize the anti-crossover activity of sgs1. PLoS Genet 2:e155

Kauppi L, Barchi M, Lange J, Baudat F, Jasin M, Keeney S (2013) Numerical constraints and feedback control of double-strand breaks in mouse meiosis. Genes Dev 27:873-886
Keeney S, Giroux CN, Kleckner N (1997) Meiosis-specific DNA doublestrand breaks are catalyzed by Spo11, a member of a widely conserved protein family. Cell 88:375-384

Keeney S, Lange J, Mohibullah N (2014) Self-organization of meiotic recombination initiation: general principles and molecular pathways. Ann Rev Genet 48:187-214

Kegel A et al (2011) Chromosome length influences replication-induced topological stress. Nature 471:392-396

Laflamme G et al (2014) Structural maintenance of chromosome (SMC) proteins link microtubule stability to genome integrity. J Biol Chem 289:27418-27431

Lee KM, Nizza S, Hayes T, Bass KL, Irmisch A, Murray JM, O’Connell MJ (2007) Brc1-mediated rescue of Smc5/6 deficiency: requirement for multiple nucleases and a novel Rad18 function. Genetics 175 : $1585-1595$

Lehmann AR, Walicka M, Griffiths DJ, Murray JM, Watts FZ, McCready S, Carr AM (1995) The rad18 gene of Schizosaccharomyces pombe defines a new subgroup of the SMC superfamily involved in DNA repair. Mol Cell Biol 15:7067-7080

Lilienthal I, Kanno T, Sjogren C (2013) Inhibition of the Smc5/6 complex during meiosis perturbs joint molecule formation and resolution without significantly changing crossover or non-crossover levels. PLoS Genet 9, e1003898

Lindroos HB, Strom L, Itoh T, Katou Y, Shirahige K, Sjogren C (2006) Chromosomal association of the Smc5/6 complex reveals that it functions in differently regulated pathways. Mol Cell 22:755-767

Lynn A, Ashley T, Hassold T (2004) Variation in human meiotic recombination. Annu Rev Genomics Hum Genet 5:317-349

Lynn A, Soucek R, Borner GV (2007) ZMM proteins during meiosis: crossover artists at work. Chromosome Res 15:591-605

Matos J, Blanco MG, Maslen S, Skehel JM, West SC (2011) Regulatory control of the resolution of DNA recombination intermediates during meiosis and mitosis. Cell 147:158-172

McAleenan A et al (2012) SUMOylation of the alpha-kleisin subunit of cohesin is required for DNA damage-induced cohesion. Curr Biol 22:1564-1575

McDonald WH, Pavlova Y, Yates JR 3rd, Boddy MN (2003) Novel essential DNA repair proteins Nse1 and Nse2 are subunits of the fission yeast Smc5-Smc6 complex. J Biol Chem 278:45460-45467

Mengiste T, Revenkova E, Bechtold N, Paszkowski J (1999) An SMClike protein is required for efficient homologous recombination in Arabidopsis. EMBO J 18:4505-4512

Nitiss JL (2009) DNA topoisomerase II and its growing repertoire of biological functions. Nat Rev 9:327-337

Oakes ML, Johzuka K, Vu L, Eliason K, Nomura M (2006) Expression of rRNA genes and nucleolus formation at ectopic chromosomal sites in the yeast Saccharomyces cerevisiae. Mol Cell Biol 26:6223-6238

Oh SD, Lao JP, Taylor AF, Smith GR, Hunter N (2008) RecQ helicase, Sgs1, and XPF family endonuclease, Mus81-Mms4, resolve aberrant joint molecules during meiotic recombination. Mol Cell 31:324-336

O'Neil NJ, Martin JS, Youds JL, Ward JD, Petalcorin MI, Rose AM, Boulton SJ (2013) Joint molecule resolution requires the redundant activities of MUS-81 and XPF-1 during Caenorhabditis elegans meiosis. PLoS Genet 9, e1003582

Osman F, Dixon J, Doe CL, Whitby MC (2003) Generating crossovers by resolution of nicked Holliday junctions: a role for Mus81-Eme1 in meiosis. Mol Cell 12:761-774

Outwin EA, Irmisch A, Murray JM, O'Connell MJ (2009) Smc5-Smc6dependent removal of cohesin from mitotic chromosomes. Mol Cell Biol 29:4363-4375

Palecek J, Vidot S, Feng M, Doherty AJ, Lehmann AR (2006) The Smc5Smc6 DNA repair complex. bridging of the Smc5-Smc6 heads by the KLEISIN, Nse4, and non-Kleisin subunits. J Biol Chem 281: 36952-36959 
Payne F et al (2014) Hypomorphism in human NSMCE2 linked to primordial dwarfism and insulin resistance. J Clin Inv 124: $4028-4038$

Pebernard S, McDonald WH, Pavlova Y, Yates JR, Boddy MN (2004) Nse1, Nse2, and a novel subunit of the Smc5-Smc6 complex, Nse3, play a crucial role in meiosis. Mol Biol Cell 15:4866-4876. doi:10. 1091/mbc.E04-05-0436

Pebernard S, Wohlschlegel J, McDonald WH, Yates JR 3rd, Boddy MN (2006) The Nse5-Nse6 dimer mediates DNA repair roles of the Smc5-Smc6 complex. Mol Cell Biol 26:1617-1630

Pebernard S, Perry JJ, Tainer JA, Boddy MN (2008) Nse1 RING-like domain supports functions of the Smc5-Smc6 holocomplex in genome stability. Mol Biol Cell 19:4099-4109

Petronczki M, Siomos MF, Nasmyth K (2003) Un ménage à quatre: the molecular biology of chromosome segregation in meiosis. Cell 112: 423-440

Potts PR (2009) The Yin and Yang of the MMS21-SMC5/6 SUMO ligase complex in homologous recombination. DNA Repair 8:499-506

Potts PR, Yu H (2007) The SMC5/6 complex maintains telomere length in ALT cancer cells through SUMOylation of telomere-binding proteins. Nat Struct Mol Biol 14:581-590

Potts PR, Porteus MH, Yu H (2006) Human SMC5/6 complex promotes sister chromatid homologous recombination by recruiting the SMC1/3 cohesin complex to double-strand breaks. EMBO J 25: 3377-3388

Roy MA, D'Amours D (2011) DNA-binding properties of Smc6, a core component of the Smc5-6 DNA repair complex. Biochem Biophys Res Commun 416:80-85

Roy MA, Siddiqui N, D'Amours D (2011) Dynamic and selective DNAbinding activity of Smc5, a core component of the Smc5-Smc6 complex. Cell Cycle (Georgetown Tex 10:690-700

Schramm S et al (2011) A novel mouse synaptonemal complex protein is essential for loading of central element proteins, recombination, and fertility. PLoS Genet 7, e1002088

Sheedy DM et al (2005) Brc1-mediated DNA repair and damage tolerance. Genetics 171:457-468

Stephan AK, Kliszczak M, Dodson H, Cooley C, Morrison CG (2011) Roles of vertebrate Smc5 in sister chromatid cohesion and homologous recombinational repair. Mol Cell Biol 31:1369-1381. doi:10. 1128/MCB.00786-10

Strom L, Lindroos HB, Shirahige K, Sjogren C (2004) Postreplicative recruitment of cohesin to double-strand breaks is required for DNA repair. Mol Cell 16:1003-1015

Sugawara N, Goldfarb T, Studamire B, Alani E, Haber JE (2004) Heteroduplex rejection during single-strand annealing requires Sgs1 helicase and mismatch repair proteins Msh2 and Msh6 but not Pms1. Proc Natl Acad Sci U S A 101:9315-9320

Tapia-Alveal C, Outwin EA, Trempolec N, Dziadkowiec D, Murray JM, O'Connell MJ (2010) SMC complexes and topoisomerase II work together so that sister chromatids can work apart. Cell cycle (Georgetown Tex) 9:2065-2070

Taylor EM, Moghraby JS, Lees JH, Smit B, Moens PB, Lehmann AR (2001) Characterization of a novel human SMC heterodimer homologous to the Schizosaccharomyces pombe Rad18/Spr18 complex. Mol Biol Cell 12:1583-1594

Taylor EM, Copsey AC, Hudson JJR, Vidot S, Lehmann AR (2008) Identification of the proteins, including MAGEG1, that make up the human SMC5-6 protein complex. Mol Cell Biol 28:11971206. doi:10.1128/MCB.00767-07
Torres-Rosell J, Losada A (2011) Smc5 flies solo. Cell Cycle (Georgetown Tex 10:874-875

Torres-Rosell J, Machin F, Aragon L (2005a) Smc5-Smc6 complex preserves nucleolar integrity in S. cerevisiae. Cell cycle (Georgetown Tex 4:868-872

Torres-Rosell J, Machin F, Farmer S, Jarmuz A, Eydmann T, Dalgaard JZ, Aragon L (2005b) SMC5 and SMC6 genes are required for the segregation of repetitive chromosome regions. Nat Cell Biol 7: 412-419

Torres-Rosell J et al (2007) The Smc5-Smc6 complex and SUMO modification of Rad52 regulates recombinational repair at the ribosomal gene locus. Nat Cell Biol 9:923-931

Unal E, Arbel-Eden A, Sattler U, Shroff R, Lichten M, Haber JE, Koshland D (2004) DNA damage response pathway uses histone modification to assemble a double-strand break-specific cohesin domain. Mol Cell 16:991-1002

Verkade HM, Bugg SJ, Lindsay HD, Carr AM, O’Connell MJ (1999) Rad18 is required for DNA repair and checkpoint responses in fission yeast. Mol Biol Cell 10:2905-2918

Verver DE, van Pelt AM, Repping S, Hamer G (2013) Role for rodent Smc6 in pericentromeric heterochromatin domains during spermatogonial differentiation and meiosis Cell. Cell Death Dis 4:e749

Verver DE, Langedijk NS, Jordan PW, Repping S, Hamer G (2014) The SMC5/6 complex is involved in crucial processes during human spermatogenesis. Biol Reprod 91:22

Vignard J, Charbonnel C, Masson JY (2011) Partners apart: Smc6independent DNA binding activity of Smc5 on single-strand DNA. Cell cycle(Georgetow Tex 10:1025-1026

Watanabe K, Pacher M, Dukowic S, Schubert V, Puchta H, Schubert I (2009) The Structural Maintenance of CHROMOSOMES 5/6 complex promotes sister chromatid alignment and homologous recombination after DNA damage in Arabidopsis thaliana. Plant Cell 21: 2688-2699

Wehrkamp-Richter S, Hyppa RW, Prudden J, Smith GR, Boddy MN (2012) Meiotic DNA joint molecule resolution depends on Nse5Nse6 of the Smc5-Smc6 holocomplex. Nucleic Acids Res 40:96339646. doi:10.1093/nar/gks713

Wu N, Yu H (2012) The Smc complexes in DNA damage response. Cell Biosci 2:5

Xaver M, Huang L, Chen D, Klein F (2013) Smc5/6-mms21 prevents and eliminates inappropriate recombination intermediates in meiosis. PLoS Genet 9, e1004067

Xue X et al (2014) Restriction of replication fork regression activities by a conserved SMC complex. Mol Cell 56:436-445

Yong-Gonzales V, Hang LE, Castellucci F, Branzei D, Zhao X (2012) The Smc5-Smc6 complex regulates recombination at centromeric regions and affects kinetochore protein sumoylation during normal growth. PLoS One 7, e51540

Youds JL, Boulton SJ (2011) The choice in meiosis - defining the factors that influence crossover or non-crossover formation. J Cell Sci 124: 501-513

Zakharyevich K, Tang S, Ma Y, Hunter N (2012) Delineation of joint molecule resolution pathways in meiosis identifies a crossoverspecific resolvase. Cell 149:334-347

Zhao X, Blobel G (2005) A SUMO ligase is part of a nuclear multiprotein complex that affects DNA repair and chromosomal organization. Proc Natl Acad Sci U S A 102:4777-4782 